\title{
REFERENCE FRAMES: DETERMINATIONS AND CONNECTIONS
}

\author{
J. O. Dickey, J. L. Fanselow, W. G. Melbourne, \\ X X Newhall, E. M. Standish, and J. G. Williams \\ Jet Propulsion Laboratory \\ California Institute of Technology \\ 4800 Oak Grove Drive \\ Pasadena, CA 91109 USA
}

\begin{abstract}
The modern space techniques have achieved such an unprecedented accuracy and precision in position measurements that there is required a rethinking and reformulation of reference frames, both in their concepts and in their realizations. Various reference frames are a natural consequence of the different observational techniques and data types. Not only are the determination and the necessity of these various frames stressed, but also their evolving nature is recognized. The current status of frame ties is highlighted, and the need for improved ties is motivated. We conclude with our concerns and recommendations.
\end{abstract}

\section{INTRODUCTION}

The advent of modern space techniques has revolutionized position measurements. These new techniques include laser ranging to the Moon and to artificial satellites; verylong-baseline interferometry (VLBI) observations of galactic and extra-galactic radio sources and of spacecraft; radio tracking of satellites; radar ranging to planets; and spacecraft tracking during planetary encounters. The accuracies of these measurements have reached an accuracy level which requires a re-examination of both the concepts and the realizations of reference frames.

The need for reference frames is seen in a variety of disciplines, such as astronomy, geodesy, celestial mechanics and geodynamics. One area in which this is a prime concern is Earth orientation (Earth rotation and polar motion) studies, a major topic of this symposium; analysis of results from the recent MERIT campaign verifies that Earth orientation is being determined with an accuracy of $\sim 5 \mathrm{~cm}$. Naturally, the goal is to have the frames themselves determined well below this accuracy level in order to optimize and combine the information content from the various complementary techniques. Frame determination and ties have a large "user community" and are crucial to a broad variety of efforts, some of which are not represented at this symposium. We should be aware of this community's needs and uses in the construction and ties of the various frames. For example, frame determination and connection are required for the upcoming Topex/Poseidon mission, designed to measure the surface topography of the global oceans at the subdecimeter level (Stewart et al., 1986). This mission will benefit from a number of the new space techniques (e.g. laser ranging, Doppler and GPS tracking) and will require optimal frame determination and ties to reach its goals.

The main thrust of this paper is to examine the necessity and motivation for the various reference frames and accurate ties between them. Section 2 discusses the 
reference frames and their determinations, while Section 3 highlights the various connections that are currently available. Prospects for the future are outlined in Section 4; our concerns and recommendations are summarized in the concluding section. The reader is referred to Dickey et al. (1986) for a review of reference frame studies at JPL/Caltech, and to Williams et al. (1983) for a more detailed account of unification of reference frames. Kovalesky (1985) and Mueller (1985) review coordinate systems.

\section{REFERENCE FRAMES AND THEIR DETERMINATIONS}

Each technique observing a particular class of objects can be expected to establish its own reference frame. Contemporary astronomy has led to the development of three principal celestial coordinate systems: the optical frame (e.g. FK4/FK5) based on the positions of galactic stars, planets, and the sun (e.g. de Vegt and Johnston,1987); the planetary/lunar ephemeris frame based on the major celestial bodies of the solar system (Standish et al., 1987); and the radio frame constructed from observations of extragalactic sources /quasars (Fanselow et al.,1984; Ma et al.,1986; and Robertson et al.,1986). It should be noted that the radio and ephemeris frames generate complementary terrestrial frames as well. Other terrestrial frames are developed through the analysis of the data from Earth-orbiting satellites [e.g. GPS (Global Positioning System), Doppler, and laser-reflecting satellites such as LAGEOS (e.g. Smith et al., 1985 and Tapley et al., 1985)]. These frames must consider local deformations as well as tectonic motion; for example, many of the laser sites are moving at rates of several $\mathrm{cm} /$ year. The celestial and terrestrial coordinate systems from a single technique and class of target are related through adopted constants and definitions. Each frame is rotated with respect to the others, and this offset may be time variable (e.g. the radio $v s$. the FK4 frame).

\section{FRAME TIES}

Measurements are inherently more accurate in their "natural" frame and hence should always be reported as such. However, to benefit from the complementarity of the various techniques, knowledge of the frame interconnections (both the rotation and the time-variable offset) is essential; these are summarized in Fig. 1 and Table 1. Recent activity in this area is indicated by the number of boxes and lines in Fig.1, entitled Connections-1986 (the accuracy cut-off here is 0.05 arcsec); a similiar figure in our earlier paper (Williams et al., 1983) had fewer boxes and connecting lines. For example, ten lines instead of the current fifteen connected the targets with the techniques, and radio stars were listed as prospects for the future. The lunar/planetary system, integrated in a joint ephemeris, is by its nature unified by the dynamics. The radio frame is tied to the ephemeris frame in several ways; one is via differential VLBI measurements of planet-orbiting spacecraft and angularly nearby quasars (Newhall et al., 1986). Another is the determination of a pulsar's position in the ephemeris frame (via timing measurements) and the radio frame [via radio interferometry (VLA) - see Backer et al., 1985]. VLA observations of the outer planets (Jupiter, Saturn, Uranus and Neptune) or their satellites provide an additional tie between these two frames (Muhleman et al., 1985). As for an optical-radio frame tie, a preliminary link has been established between the FK5 optical frame and the JPL radio reference frame via the differential VLBI measurement of optically bright radio stars and angularly nearby quasars coupled with comparisons of their optical positions (see Lestrade et al., 1987), and also by the use of the optical positions of quasars (Purcell, 1979). The optical and ephemeris frames are tied by optical observations of the planets. 


\title{
TABLE 1 FRAME TIES
}

\author{
LUNAR/PLANETARY EPHEMERIS \\ * COUPLED DYNAMICS \\ * FUNDAMENTAL ZERO POINT. DYNAMICAL EQUINOX
}

\author{
VLBI/EPHEMERIS \\ * $\triangle$ VLBI \\ * MILLISECOND PULSAR \\ * VLA MEASUREMENTS
}

\author{
OPTICAL/RADIO \\ * OPTICAL-RADIO STARS \\ * "JOINT" PLANETARY MEASUREMENTS
}

\author{
OPTICAL/EPHEMERIS \\ * occultations \\ * OPTICAL PLANETARY OBSERVATION
}

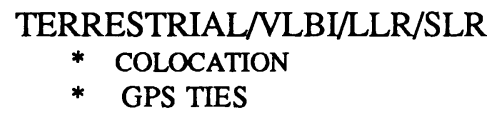

\section{PROSPECTS FOR THE FUTURE}

The future is promising with on-going and planned efforts in several areas. For optical astrometry, Hipparcos will measure a network of stars over the entire sky with accuracies of $\sim 2$ mas (Kovalevsky, 1980), while the Space Telescope will measure small fields with similar differential accuracy. However, the Space Telescope can observe much fainter objects (Jefferys, 1980) including the optical counterparts of extragalactic radio sources, all but possibly one of which are too faint for Hipparcos. A joint program would produce an accurate stellar network linked to the quasar radio frame by the Space Telescope. The occultations of stars by planets and planetary rings can provide an additional link between the optical and ephemeris frames. Also, optical interferometry offers exciting possibilities with the potential resolution being two or three orders of magnitude finer than that of VLBI (Reasenberg, 1986). Improved ephemeris-radio frame ties can be accomplished by VLBI observations of pulsars, additional VLA observations of the outer planets and satellites, and future differential VLBI experiments (such as that with spacecraft orbiting Jupiter and Saturn). Additional VLA observations of Neptune are now planned for January 1987 (D. Jones, private communication, 1986). The millisecond pulsar PSR1937+214, having a period of 1.6 msec, has exceptionally low timing noise. Its position in the ephemeris frame can be measured to $\sim 1$ mas. This will allow a radio-planetary frame tie, limited only by the accuracy of interferometric position measurements. A factor of about five improvement (down to 0.01") is expected here with the full implementation of VLBI observations (Linfield, private communication, 1986). An initial experiment of this type is planned by $\mathrm{R}$. Linfield and C. Gwinn. Turning to the terrestrial-frame techniques, colocation provides direct links between the systems involved. GPS also furnishes an alternate method for terrestrial ties. A continuing program is required not only to maintain the terrestrial frames and their ties, but to monitor tectonic motion and local deformations 


\section{CONNECTIONS}

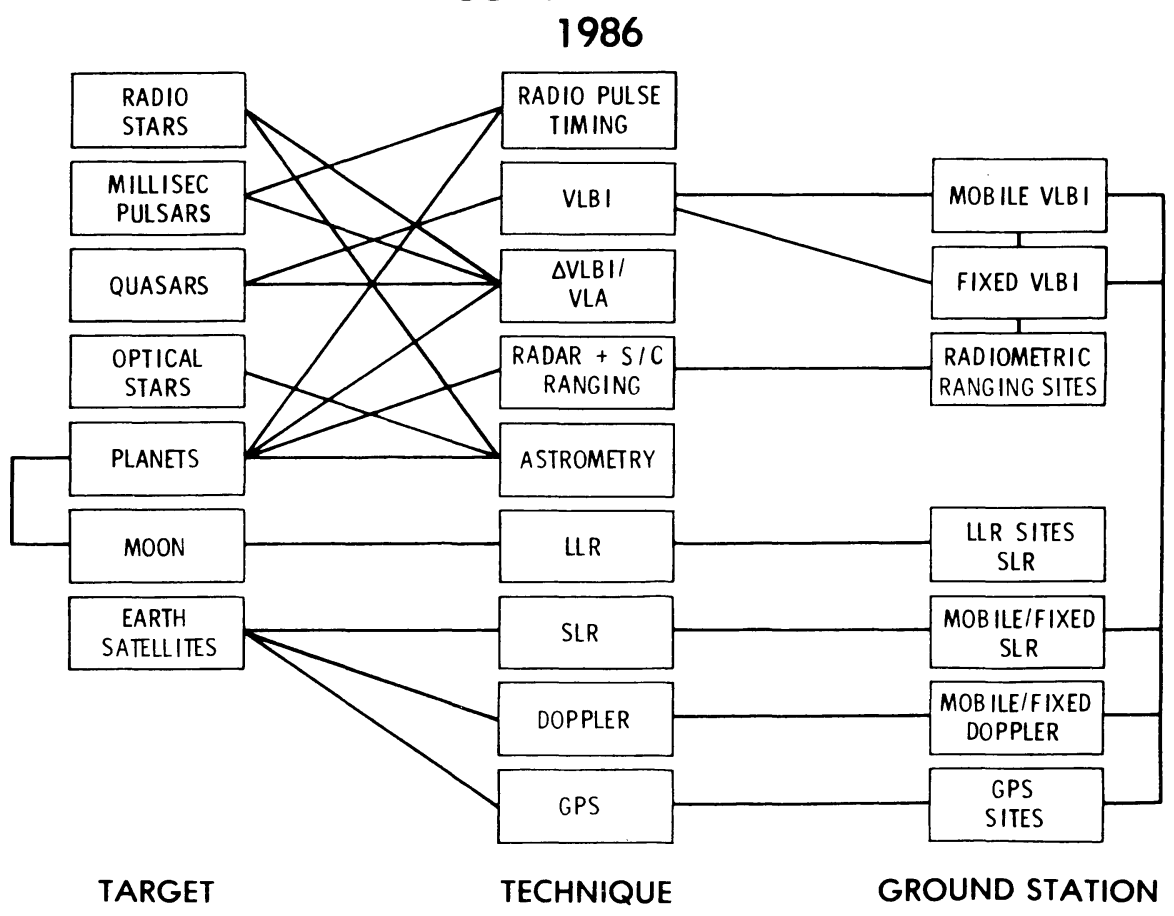

Fig. 1

such as ocean loading. Clearly in years to come Fig. 1 will become even richer in the number and accuracies of connections.

\section{CONCERNS AND RECOMMENDATIONS}

The concept and the realization of reference frames are clearly needed for a variety of efforts: astronomy, geodesy and geodynamics, to list a few. Reference frames are the standard scale against which measurements are made. The current space techniques have achieved remarkable accuracies; however, continued improvements and other new techniques may achieve even higher accuracy. From Fig. 1 it is evident that no one technique can do "all things for all people." To advocate a single frame, whose definition is time invariant, is not an appropriate or realistic goal. The transient nature of these frames, in both concept and realization, and their evolving nature should be appreciated. We recommend that the community recognize the necessity of different frames, their transitory nature, and the need for improvements and the constant maintenance of their interconnections. 
Acknowledgment: This paper presents the results of one phase of research carried out at the Jet Propulsion Laboratory, California Institute of Technology, sponsored by the National Aeronautics and Space Administration.

\section{References:}

Backer, D. D., Fomalont, E. B., Goss, W. M., Taylor, J. H. and Weisberg, J. M., "Accurate Timing and Interferometric Positions for the Millisecond Pulsar 1937+21 and the Binary Pulsar 1913+16", Astron. J., 90, 2275, 1985.

de Vegt, C., and Johnston, K., "Fundamental Reference Frames", this volume, 1987.

Dickey, J. O., Esposito, P.B., Lestrade, J.-F., Linfield, R.P., Melbourne, W. G. , Newhall, X X, Niell, A. E. , Preston, R. A., Standish, E. M., Williams, J. G., Muhleman, D.O., Berge, G. L., and Rudy, D. J., "Reference Frame Studies at JPL/Caltech", presented at the Joint Discussion on Reference Frames at the XIXth General Assembly of the International Astronomical Union (New Dehli, India November 1985), Highlights of Astronomy, 7., ed. J.-P. Swings, Reidel, Dordrecht-Holland, 93-96, 1986.

Fanselow, J. L., Sovers, O. J., Thomas, J. B., Purcell, G. H., Cohen, E. J., Rogstad, D.H., Skjerve, L. J., and Spitzmesser, D. J., "Radio Interferometric

Determination of Source Positions Utilizing Deep Space Antennas, 1971 to 1980", Astron. J., 89, 987-998, 1984.

Jefferys, W. H., "Astrometry with the Space Telescope", Celestial Mech., 22, 175$181,1980$.

Kovalevsky, J., "Global Astrometry by Space Techniques", Celestial Mech., 22, 153 $163,1980$.

Kovalevsky, J., "Systèmes de Référence Terrestres et Célestes", Bulletin Astronomique, 10, 2, 87-93, 1985.

Lestrade, J.-F., Requieme, Y., Rapaport, M., and Preston, R. A., "Preliminary Relationship between the FK5 and the JPL Radio Reference Frames", this volume, 1987.

Ma, C., Clark, T. A., Ryan, J. W., Herring, T. A., Shapiro, I. I., Corey, B. E., Hinteregger, H. F., Rogers, A. E. E., Whitney, A. R., Knight, C. A., Lundquist, G. L., Shaffer, D. B., Vandenburg, N. R., Pigg, J. C., Schupler, B. R., and Ronnang, B. O., "Radio-Source Positions from VLBI", Astron. J., 92, 1020-1029, 1986.

Muhleman, D. O., Berge, G. L., Rudy, D. J., Niell, A. E., Linfield, R. P., and Standish, E. M., "Precise Position Measurements of Jupiter, Saturn and Uranus Systems with the Very Large Array", Celestial Mech., 37, 329-337, 1985.

Mueller, I. I, "Reference Coordinate Systems and Frames: Concepts and Realizations", Bull. Géod., 59, 181-188, 1985. 
Newhall, X X, Preston, R. A., and Esposito, P. B., "Relating the JPL VLBI Reference Frame and the Planetary Ephemerides", Proceedings of the International Astronomical Union Symposium 109: Astrometric Techniques, (Gainesville, Florida, 1984), H. K. Eichhorn and R. J. Leacock, eds., Reidel, DordrechtHolland, 789-794, 1986.

Purcell, G. H., Cohen, E. J., Fanselow, J. L., Rogstad, D. H., Skjerve, L. J., Spitzmesser, D. J., Thomas, J. B., "Current Results and Developments in Astrometric VLBI at the Jet Propulsion Laboratory", Proceedings of the International Astronomical Union Colloquium No. 48: Modern Astrometry, F. V. Prochazka and R.H. Tucker, eds., published by the University Observatory, Vienna; Herausgeber and Verleger: Institut für Astronomie (UniversitätsSternwarte Wien), 185-194, 1979.

Reasenberg, R. D., "Microarcsecond Astrometric Interferometry", Proceedings of the International Astronomical Union Symposium 109: Astrometric Techniques, (Gainesville, Florida, 1984), H. K. Eichhorn and R. J. Leacock, eds., Reidel, Dordrecht-Holland, 1986.

Robertson, D. S., Fallon, F. W., and Carter, W. E., "Celestial Reference Coordinate Systems - Submilliarcsec Precision Demonstrated with VLBI Observations", Astron. J., 91, 1456-1462, 1986.

Smith, D. E., Christodoulidis, D. C., Kolenkiewicz, R., Dunn, P. J., Klosko, S. M., Torrence, M. H., Fricke, S., and Blackwell, S., "A Global Geodetic Reference Frame from LAGEOS Ranging", J. Geophys. Res., 90, B11, 9221-9234, 1985.

Stewart, R., Fu, L.-L., Lefebvre, M., "Science Opportunities From the Topex/Poseidon Mission", JPL Publication 86-18, 1986

Standish, E. M., Newhall, X X, Williams, J. G., Dickey, J. O., "The Reference Frame of the Ephemerides", this volume, 1987.

Tapley, B.D., Schutz, B. E., and Eanes, R. J., "Station Coordinates, Baselines, and Earth Rotation from LAGEOS Laser Ranging: 1976-1984", J. Geophys. Res., 90, B11, 9235-9248, 1985.

Williams, J. G., Dickey, J. O., Melbourne, W. G., and Standish, E. M., "Unification of Celestial and Terrestrial Coordinate Systems", Proceedings of the International Association of Geodesy (IAG) Symposia, International Union of Geodesy and Geophysics (IUGG) XVIIIth General Assembly, Hamburg, FRG, August 15-27, 1983, Ohio State University, Dept. of Geodetic Science and Surveying, Columbus, Ohio 43210, Vol. 2, 12-27, 1983. 


\section{DISCUSSION}

Bauersima: The purpose of a Terrestrial Reference Frame is to categorize the configuration of terrestrial points as functions of time in a homogenous manner. But only such coordinate invariant relations as distances and angles makes physical sense. Therefore, to tie different reference frames together is a question of solving the observation equations of different methods together, using the same 6 kinematical conditions (e.g. no translation, no rotation, in the mean).

Reply by Dickey: You have outlined some of the concepts involved. However, concept and realization are not necessarily the same. The realization of these concepts is challenging.

T. Fukushima: Japan has launched a new type of satellite for geodesic use in Aug. 1986. It has two functions: to reflect laser light, and to reflect sunlight. The highest magnitude of this satellite is 1.5 and it flashes 2 times per second. Use of this satellite enables us to find the relation between satellite RF's and optical RF's.

Reply by Dickey: Thank you for your comments. Some of us heard a very interesting presentation concerning this at the NASA Crustal Dynamics Meeting last week.

A. Niell: We have begun ranging measurements to Lageos with the Haystack Observatory Long Range Imaging Radar. The accuracy should be comparable to that of the lasers, i.e. a few centimeters rms.

Reply by Dickey: We will be looking forward to your results.

S. Débarbat: You conclude your presentation with a list of recommendations. To whom are these addressed?

Reply by Dickey: We hope to have a resolution passed at this meeting concerning these issues.

T. Clark: One other possibility for tying reference frames together : monostatic \& bistatic radar tracking of satellites like Lageos and Ajisai could be done from sites which also produce quasar VLBI data. Monostatic measurement would yield precise spacecraft ranging data, while bistatic measurements (using VLBI techniques) could produce milliarcsecond angular positions of the satellites.

Reply by Dickey: This would be an interesting development.

Hughes: A few items. First: you should add a line between "Astrometry" and "Quasars" on your wiring diagram. Otherwise you ignore a great deal of effort by many people. Second: You characterized the optical frame as "stars" only and then used optical observations of planets only as a tie mechanism between an ephemeris frame and the optical frame. Without the planetary observations, there wouldn't be an optical frame. Also, the very important observations of the Sun should be included. Third: I wish you would include optical and IR interferometry with Hipparcos and Space Telescope since these techniques are really coming along. Finally, I would mention a coordinated reference frame some of us are starting on which will tie optical and radio frames on a global basis (i.e., includes the southern hemisphere.)

Reply by Dickey: Thank you for your comments.

1) One of the purposes in showing such a diagram is to get people thinking of further connections. It looks like we have succeeded. We have not included all possible connections but instead imposed an accuracy requirement. An optimistic accuracy limit on the optical measurement of radio stars is 50 mas. Optical measurement of quasars would be less accurate.

2) I agree that the Sun and planets should be included under the category of targets for optical astrometry.

3) We did not include Hipparcos and Space Telescope since these are future developments as we outlined in recommendations for the future. This wiring diagram (connections, 1986) is meant to represent the current status and does not project into the future. 